\title{
ON THE GENERALIZED HEAT-EQUATION
}

\author{
by C. NASIM and B. D. AGGARWALA
}

(Received 18th September 1983)

\section{Introduction}

The general heat equation is defined as

$$
\frac{\partial^{2} u}{\partial x^{2}}+\frac{2 v}{x} \frac{\partial u}{\partial x}-\frac{\alpha^{2}}{x^{2}} u=\frac{\partial u}{\partial t}
$$

or

$$
\Delta_{x} u=\frac{\partial u}{\partial t}, \quad \Delta_{x} \equiv \frac{\partial^{2}}{\partial x^{2}}+\frac{2 v}{x} \frac{\partial}{\partial x}-\frac{\alpha^{2}}{x^{2}}
$$

where $v$ is a fixed positive number and $\alpha$ is a fixed number. If $v=\alpha=0$, then (1.1) reduces to the ordinary heat equation

$$
\frac{\partial^{2} u}{\partial x^{2}}=\frac{\partial u}{\partial t}
$$

where $u(x, t)$ is regarded as the temperature at a point $x$ at time $t$, in an infinite insulated rod extended along the $x$-axis in the $x t$-plane. If we set $v=\frac{1}{2}$, then (1.1) becomes

$$
\frac{\partial^{2} F}{\partial x^{2}}+\frac{\partial^{2} F}{\partial y^{2}}=\frac{\partial F}{\partial t}
$$

the heat equation in two dimensions, where the solutions are of the type

$$
F(x, y ; t)=u(r, t) \sin \alpha \theta,
$$

in polar coordinates; and represents the temperature in a plane sector of angle $\pi / \alpha$.

Further, if we put $v=1$ and $\alpha^{2}=n(n+1)$, then (1.1), yields the heat equation in three dimensions

$$
\frac{\partial^{2} F}{\partial x^{2}}+\frac{\partial^{2} F}{\partial y^{2}}+\frac{\partial^{2} F}{\partial z^{2}}=\frac{\partial F}{\partial t}
$$


where the solutions are of the form

$$
F(x, y, z ; t)=u(r, t) P_{n}(\cos \phi)
$$

in spherical coordinates representing the temperature in a cone of angle $\phi$. Here $P_{n}(z)$ are the Legendre polynomials. Consequently, the heat equation (1.1) can be regarded as representing a general situation for the flow of heat.

The object of this paper is to study the analytic consequences of the general heat equation. We shall devote our main effort towards establishing some properties of the source solution and an algorithm for the inversion of the heat transform. The case $\alpha=0$ has been dealt with thoroughly in [1].

\section{The source solution}

Consider the temperature at $x=\xi$, as instantaneously enormous at $t=0^{+}$but levelling off rapidly. Thus there is a source at $x=\xi$; and the temperature function is now defined as the source solution. To find the source solution $u(\xi, x, t)$ of $(1.1)$, we consider the equation

$$
\frac{\partial^{2} u}{\partial x^{2}}+\frac{2 v}{x} \frac{\partial u}{\partial x}-\frac{\alpha^{2}}{x^{2}} u=\frac{\partial u}{\partial t}-\delta(x-\xi) \delta(t)
$$

where $\delta$ is the Dirac delta function. If

$$
\bar{u}=\int_{0}^{\infty} u(x, t) e^{-s t} d t
$$

then (2.1) gives,

$$
\frac{\partial^{2} \bar{u}}{\partial x^{2}}+\frac{2 v}{x} \frac{\partial \bar{u}}{\partial x}-\frac{\alpha^{2}}{x^{2}} \bar{u}=s \bar{u}+\delta(x-\xi) .
$$

The solution, then, is

$$
\bar{u}= \begin{cases}\xi^{v+\frac{1}{2}} x^{\frac{1}{2}-v} K_{\mu}\left(s^{\frac{1}{2}} \xi\right) I_{\mu}\left(s^{\frac{1}{2}} x\right), & x<\xi \\ \xi^{v+\frac{1}{2}} x^{\frac{1}{2}-v} I_{\mu}\left(s^{\frac{1}{2}} \xi\right) K_{\mu}\left(s^{\frac{1}{2}} x\right), & x>\xi\end{cases}
$$

and by the inverse Laplace transform, [2, p. 284],

$$
U \equiv U(\xi, x ; t)=\xi^{2 v} G_{\mu}(\xi, x ; t),
$$

where

$$
G_{\mu}(\xi, x ; t)=\frac{1}{2 t}(\xi x)^{\frac{1}{2}-v} e^{-\left(\xi^{2}+x^{2}\right) / 4 t} I_{\mu}\left(\frac{\xi x}{2 t}\right)=G(\xi, x ; t),
$$


say, and $\mu^{2}=\left(v-\frac{1}{2}\right)^{2}+\alpha^{2}, \mu>-1, t>0$. We shall call the function $U$ to be the source solution of the general heat equation (1.1). And for simplicity we shall say that

$$
U(\xi, x ; t) \in H .
$$

Next we shall discuss some of the more interesting properties of the source solution and in particular, the so-called Green's function $G(\xi, x ; t)$. We note that

$$
G(\xi, x ; t)=(\xi x)^{\frac{1}{2}-v} \int_{0}^{\infty} u e^{-t u^{2}} J_{\mu}(\xi u) J_{\mu}(x u) d u
$$

where $\mu>-1, t>0,[3$, p. 51]. As a direct result of the definition of the function $U(\xi, x ; t)$, we have the following theorem.

Theorem 2.1. Let $U(\xi, x ; t)$ be as defined above. Then

(i) $U(\xi, x ; t)>0, \quad \xi, x>0$,

(ii) $U\left(\lambda \xi, \lambda x ; \lambda^{2} t\right)=U(\xi, x ; t)$,

(iii) $x^{2 v} U(\xi, x ; t)=\xi^{2 v} U(x, \xi ; t)$.

Theorem 2.2. Let $U(\xi, x ; t)$ be as defined above. Then

(i) $\int_{0}^{\infty} \xi^{k} U(\xi, x ; t) d \xi=x^{k}$,

(ii) $|U(\xi, s ; t)| \leqq A(2 t)^{-\frac{1}{2}}|\xi|^{v}\left(\sigma^{2}+\tau^{2}\right)^{-\frac{1}{2} v} e^{-\left((\sigma-\xi)^{2}+\tau^{2}\right) / 4 t}$,

where $s=\sigma+i \tau, \sigma>0,-\infty<\tau<\infty$,

(iii) $\left|\frac{\partial}{\partial s} U(\xi, s ; t)\right| \leqq B t^{-1}|\xi|^{v}\left(\sigma^{2}+\tau^{2}\right)^{-\frac{1}{2} v} e^{-\left\{(\sigma-\xi)^{2}+\tau^{2}\right\} / 4 t}$

$$
\times\left(\frac{\frac{1}{2}-v+\mu}{\sqrt{\sigma^{2}+\tau^{2}}}+\frac{\sqrt{\sigma^{2}+\tau^{2}}}{2 t}+\frac{\xi}{2 t}\right)
$$

(iv) $\left|\frac{\partial}{\partial t} U(\xi, x ; t)\right| \leqq C t^{-3 / 2}\left(\frac{\xi}{x}\right)^{v} e^{-(\xi-x)^{2} / 4 t}\left(\mu-1+\frac{(\xi-x)^{2}}{4 t}\right)$.

Proof. Conclusion (i) follows by direct computation. From the definition given in (2.2) and the asymptotic behaviour $I_{v}(z) \sim(1 / \sqrt{2 \pi z}) e^{z}$, we have

$$
U(\xi, s ; t)=\frac{\xi^{2 v}}{2 t}(\xi s)^{\frac{1}{2}-v} e^{-\left(\xi^{2}+s^{2}\right) / 4 t} I_{\mu}\left(\frac{\xi s}{2 t}\right),
$$

and it follows that

$$
|U(\xi, s ; t)| \leqq A(2 t)^{-\frac{1}{t}}|\xi|^{v}\left(\sigma^{2}+\tau^{2}\right)^{-\frac{1}{t} v} e^{-\left\{(\sigma-\xi)^{2}+\tau^{2}\right\} / 4 t} .
$$


Also,

$$
\begin{aligned}
\frac{\partial}{\partial s} U(\xi, s ; t)= & \frac{\partial}{\partial s}\left\{\frac{\xi^{2 v}}{2 t}(\xi s)^{\frac{1}{2}-v} e^{-\left(\xi^{2}+s^{2}\right) / 4 t} I_{\mu}\left(\frac{\xi s}{2 t}\right)\right\} \\
= & \frac{\xi^{\frac{1}{2}+v+\mu}}{(2 t)^{\mu+1}} e^{-\xi^{2} / 4 t} \frac{\partial}{\partial s}\left\{s^{\frac{1}{2}-v+\mu} e^{-s^{2} / 4 t}\left(\frac{\xi s}{2 t}\right)^{-\mu} I_{\mu}\left(\frac{\xi s}{2 t}\right)\right\} \\
= & \frac{1}{2 t} \xi^{\frac{1}{2}+v} e^{-\left(\xi^{2}+s^{2}\right) / 4 t}\left\{\left(\frac{1}{2}-v+\mu\right) s^{-\frac{1}{2}-v} I_{\mu}\left(\frac{\xi s}{2 t}\right)\right. \\
& \left.-\frac{s^{3 / 2-v}}{2 t} I_{\mu}\left(\frac{\xi s}{2 t}\right)+\frac{\xi}{2 t} s^{\frac{1}{2}-v} I_{\mu+1}\left(\frac{\xi s}{2 t}\right)\right\} \\
\sim & \frac{1}{(2 t)^{\frac{1}{2}}}\left(\frac{\xi}{s}\right)^{v} e^{-(\xi-s)^{2} / 4 t}\left[\left(\frac{1}{2}-v+\mu\right) \frac{1}{S}+\frac{s}{2 t}+\frac{\xi}{2 t}\right]
\end{aligned}
$$

thus,

$$
\begin{aligned}
\left|\frac{\partial}{\partial s} U(\xi, s ; t)\right| \leqq \frac{|\xi|^{\nu}}{(2 t)^{\frac{1}{2}}}\left(\sigma^{2}+\tau^{2}\right)^{-\frac{1}{2} v} e^{-\left\{(\sigma-\xi)^{2}+\tau^{2}\right\} / 4 t} \\
\cdot\left[\frac{\frac{1}{2}-v+\mu}{\sqrt{\sigma^{2}+t^{2}}}+\frac{\sqrt{\sigma^{2}+t^{2}}}{2 t}+\frac{\xi}{2 t}\right]
\end{aligned}
$$

proving the assertion (iii). To prove the assertion (iv), by direct computation, we have

$$
\frac{\partial}{\partial t} U(\xi, x ; t)=\frac{1}{4 t^{2}} U(\xi, x ; t)\left(x^{2}+\xi^{2}-4 t+4 \mu t^{2}\right)-\frac{\xi x}{2 t^{2}} U_{\mu-1}(\xi, x ; t)
$$

hence

$$
\left|\frac{\partial}{\partial t} U(\xi, x ; t)\right| \leqq C t^{-3 / 2}\left(\frac{\xi}{x}\right)^{v} e^{-(\xi-x)^{2} / 4 t}\left\{\mu-1+\frac{(\xi-x)^{2}}{4 t}\right\}
$$

as required.

Theorem 2.3. If $0 \leqq x<\infty, 0 \leqq y<\infty$ and $0<t_{1}<t_{2}$, then

(i) $\int_{0}^{\infty} U(\xi, x ; t) G\left(\xi, y ; t_{2}\right) d \xi=G\left(x, y ; t_{1}+t_{2}\right)$,

(ii) $\int_{0}^{\infty} U\left(\xi, i x ; t_{1}\right) G\left(i \xi, y ; t_{2}\right) d \xi=(-1)^{k} G\left(x, y ; t_{2}-t_{1}\right)$

where

$$
k=\mu-v+\frac{1}{2} \text { and } \mu>-1 .
$$

Proof. By using the estimates derived in the last theorem, it is easy to show that the integrals in the assertions (i) and (ii) above exist. Now, by direct evaluation, [2, p. 197]. 


$$
\begin{aligned}
& \int_{0}^{\infty} U\left(\xi, x ; t_{1}\right) G\left(\xi, y ; t_{2}\right) d \xi \\
& =\frac{(x y)^{\frac{1}{2}-v}}{4 t_{1} t_{2}} e^{-\left(x^{2} / t_{1}+y^{2} / t_{2}\right) / 4} \int_{0}^{\infty} I_{\mu}\left(\frac{\xi x}{2 t_{1}}\right) I_{\mu}\left(\frac{\xi y}{2 t_{2}}\right) e^{-\xi^{2}\left(1 / t_{1}+1 / t_{2}\right) / 4} \xi d \xi \\
& =\frac{1}{2\left(t_{1}+t_{2}\right)}(x y)^{\frac{1}{2}-v} e^{-\left(x^{2}+y^{2}\right) / 4\left(t_{1}+t_{2}\right)} I_{\mu}\left(\frac{x y}{2\left(t_{1}+t_{2}\right)}\right)=G\left(x, y ; t_{1}+t_{2}\right)
\end{aligned}
$$

as required. Also, the assertion (ii) can, similarly, be established. Note that assertion (ii) can be considered as the inversion of the integral equation in (i).

\section{The heat transform}

If we now consider the source solution $U$ as the kernel, then for a suitable $f$, its heat transform $F$ is defined by

$$
x^{k} F(x, t)=\int_{0}^{\infty} U(\xi, x ; t) \xi^{k} f(\xi) d \xi
$$

where $k=\mu-v+\frac{1}{2}, \mu^{2}=\left(v-\frac{1}{2}\right)^{2}+\alpha^{2}$ and $\mu>-1$.

Theorem 3.1. If $f(x)$ is bounded and continuous in $0<x<\infty$, and has a heat transform $F(x, t)$, then $x^{k} F(x, t) \in H, t>0$, where $k=\mu-v+\frac{1}{2}, \mu>-1$.

Proof. From (3.1) above,

$$
\begin{aligned}
\left|x^{k} F(x, t)\right| & \leqq A \int_{0}^{\infty} U(\xi, x ; t) \xi^{k} d \xi \\
& <B t^{-\frac{1}{2}} x^{-v} \int_{0}^{\infty} \xi^{v+k} e^{-(\xi-x)^{2} / 4 t} d \xi<\infty,
\end{aligned}
$$

using the estimate (2.4), where $A=u . b . f(x), 0<x<\infty$. Hence the integral defining the function $F$ exists and is in fact absolutely convergent. Now

$$
\begin{aligned}
\Delta_{x}\left[x^{k} F(x, t)\right] & =\Delta_{x}\left[\int_{0}^{\infty} U(\xi, x ; t) \xi^{k} f(\xi) d \xi\right] \\
& =\int_{0}^{\infty} \Delta_{x}[U(\xi, x ; t)] \xi^{k} f(\xi) d \xi \\
& =\int_{0}^{\infty} \frac{\partial}{\partial t} U(\xi, x ; t) \xi^{k} f(\xi) d \xi \\
& =\frac{\partial}{\partial t} \int_{0}^{\infty} U(\xi, x ; t) \xi^{k} f(\xi) d \xi \\
& =\frac{\partial}{\partial t}\left[x^{k} F(x, t)\right],
\end{aligned}
$$


proving that $x^{k} F(x, t)$ satisfies the heat equation (1.1), hence $x^{k} F(x, t) \in H$, provided one can justify interchanging the operators $\Delta_{x}$ and $\partial / \partial t$ with the integral sign. Now, using (2.6), we have

$$
\begin{aligned}
\int_{0}^{\infty}\left|\Delta_{x}[U(\xi, x ; t)] \xi^{k} f(\xi)\right| d \xi & =\int_{0}^{\infty}\left|\frac{\partial}{\partial t}[U(\xi, x ; t)] \xi^{k} f(\xi)\right| d \xi \\
& \leqq A(x, t) \int_{0}^{\infty} \xi^{k+v} e^{-(\xi-x)^{2} / 4 t}|f(\xi)| d \xi \\
& \leqq B(x, t) \int_{0}^{\infty} \xi^{k+v} e^{-\xi^{2} / 8 t}|f(\xi)| d \xi<\infty
\end{aligned}
$$

for all $x>0$ and $t>0$, and making use of the fact that

$$
(\xi-x)^{2} \geqq \frac{1}{2} \xi^{2}-x^{2}
$$

hence

$$
e^{-(\xi-x)^{2} / 4 t} \leqq e^{-\xi^{2} / 8 t+x^{2} / 4 t}
$$

giving us the desired justification.

Theorem 3.2. If $f(x)$ is bounded and (nntinuous in $0<x<\infty$, then

(i) $s^{k} F(s, t)=\int_{0}^{\infty} U(\xi, s ; t) \xi^{k} f(\xi) d \xi$,

$$
s=\sigma+i \tau, \quad \sigma>0, \quad-\infty<\tau<\infty, \quad \text { and }
$$

(ii) $s^{k} F(s, t)$ is analytic in the complex half plane $\operatorname{Re} s>0$.

Proof.

$$
\begin{aligned}
\left|s^{k} F(s, t)\right| & \leqq \int_{0}^{\infty}\left|U(\xi, s ; t) \xi^{k} f(\xi)\right| d \xi \\
& \leqq A(\sigma, \tau, t) \int_{0}^{\infty} \xi^{k+v} e^{-(\xi-\sigma)^{2} / 4 t}|f(\xi)| d \xi<\infty,
\end{aligned}
$$

due to (2.4). Hence the function $s^{k} F(s, t)$ exists and is defined by a uniformly convergent integral for $\sigma>0$ and $t>0$. To prove that $s^{k} F(s, t)$ is analytic in the complex half plane $\sigma>0$, we need to show that

$$
\int_{0}^{\infty} \frac{\partial}{\partial s} U(\xi, s ; t) \xi^{k} f(\xi) d \xi
$$


converges uniformly. Now, using (2.5) we have

$$
\begin{aligned}
\int_{0}^{\infty}\left|\frac{\partial}{\partial s} U(\xi, s ; t) \xi^{k} f(\xi)\right| d \xi & \leqq B(\sigma, \tau, t) \\
& \times \int_{0}^{\infty} \xi^{v+k} e^{-(\xi-\sigma)^{2} / 4 t}|f(\xi)| d \xi
\end{aligned}
$$

which converges uniformly for all $\sigma>0$ and $t>0$ as seen above. Hence the theorem.

Corollary. $\left|s^{k} F(s, t)\right| \leqq C\left(\sigma^{2}+\tau^{2}\right)^{\frac{1}{2}-\frac{1}{2} v} e^{-\left(\sigma^{2}+\tau^{2}\right) / 4 t}$.

Theorem 3.3. Let $F(x, t)$ be as defined above. Then

$$
x^{k} F\left(x, t+t_{1}\right)=\int_{0}^{\infty} U(\xi, x ; t) \xi^{k} F\left(\xi, t_{1}\right) d \xi
$$

for a fixed $t_{1}>0$.

Proof. It is easy to see that the integral in (3.4) exists. Now,

$$
\begin{aligned}
\int_{0}^{\infty} U(\xi, x ; t) \xi^{k} F\left(\xi, t_{1}\right) d \xi & =\int_{0}^{\infty} U(\xi, x ; t) d \xi \int_{0}^{\infty} U\left(y, \xi ; t_{1}\right) y^{k} f(y) d y \\
& =\int_{0}^{\infty} y^{k} f(y) d y \int_{0}^{\infty} U(\xi, x ; t) U\left(y, \xi ; t_{1}\right) d \xi \\
& =\int_{0}^{\infty} y^{k+2 v} f(y) d y \int_{0}^{\infty} U(\xi, x ; t) G\left(y, \xi ; t_{1}\right) d \xi \\
& =\int_{0}^{\infty} y^{k+2 v} f(y) G\left(x, y ; t+t_{1}\right) d y \\
& =\int_{0}^{\infty} U\left(y, x ; t+t_{1}\right) y^{k} f(y) d y \\
& =x^{k} F\left(x, t+t_{1}\right),
\end{aligned}
$$

making use of the result of Theorem 2.3 and the equation (3.1).

The change of order of integration in the above analysis is justified due to absolute convergence. This establishes the equation (3.4).

Corollary. If $t_{1} \rightarrow 0$, then (3.4) yields the heat transform (3.1), formally. 


\section{The inversion}

In this section we shall find the inversion of the integral equation (3.4), and then deduce the inversion of the heat transform (3.1).

Theorem 4.1. Let $F(x, t)$ be defined as above. If

$$
x^{k} F\left(x, t+t_{1}\right)=\int_{0}^{\infty} U(\xi, x ; t) \xi^{k} F\left(\xi, t_{1}\right) d \xi
$$

then

$$
x^{k} F\left(x, t_{1}\right)=\int_{0}^{\infty} U(\xi, i x ; t)\left(\frac{\xi}{i}\right)^{k} F\left(i \xi, t+t_{1}\right) d \xi
$$

where $t, t_{1}, v>0, \mu>-1$ and $k=\mu-v+\frac{1}{2}$.

Proof. Now from (3.4),

$$
(i x)^{k} F\left(i x, t+t_{1}\right)=\int_{0}^{\infty} U(\xi, i x ; t) \xi^{k} F\left(\xi, t_{1}\right) d \xi
$$

or simplifying,

$$
x^{\nu+k-\frac{1}{2}} F\left(i x, t+t_{1}\right)=\frac{1}{2 t} \int_{0}^{\infty}(\xi)^{\nu+k+\frac{1}{2}} e^{-\left(\xi^{2}-x^{2}\right) / 4 t} J_{\mu}\left(\frac{\xi x}{2 t}\right) F\left(\xi, t_{1}\right) d \xi
$$

If we put $x=2 t y$, then the above equation gives,

$$
(2 t)^{k+v+\frac{1}{2}} y^{k+v} e^{-y^{2} t} F\left(i 2 t y, t+t_{1}\right)=\int_{0}^{\infty} \sqrt{\xi y} J_{\mu}(\xi y) \xi^{k+v} F\left(\xi, t_{1}\right) e^{-\xi^{2} / 4 t} e d \xi
$$

which is the usual form of the Hankel transform, and therefore on inverting gives

$$
y^{k+v} e^{-y^{2} / 4 t} F\left(y, t_{1}\right)=\int_{0}^{\infty} \sqrt{\xi y} J_{\mu}(\xi y)(2 t)^{k+v+\frac{1}{2}} e^{-\xi^{2} t} F\left(i 2 t \xi, t+t_{1}\right) \xi^{k+v} d \xi
$$

Again, let $v=2 t \xi$, and simplify to get

$$
y^{k} F\left(y, t_{1}\right)=\int_{0}^{\infty} U(v, i y ; t)\left(\frac{v}{i}\right)^{k} F\left(i v, t+t_{1}\right) d v,
$$

Corollary 1. (3.4) can alternatively be written as

$$
y^{k} F\left(y, t_{1}\right)=\int_{0}^{i \infty} U(x, y ;-t) x^{k} F\left(x, t+t_{1}\right) d x
$$


Corollary 2. Let $t_{1} \rightarrow 0$. Then

$$
\lim _{t_{1} \rightarrow 0} F\left(x, t_{1}\right)=f(x)
$$

and the pair of heat transforms (3.4) and (3.5) reduce to, respectively,

$$
x^{k} F(x, t)=\int_{0}^{\infty} U(\xi, x ; t) \xi^{k} f(\xi) d \xi
$$

and

$$
x^{k} f(x)=\int_{0}^{\infty} U(\xi, i x ; t)\left(\frac{\xi}{i}\right)^{k} F(i x, t) d \xi
$$

formally.

\section{Operational calculus}

By Taylor series expansion, we have

$$
\begin{aligned}
x^{k} F\left(x, t+t_{1}\right) & =x^{k} \sum_{n=0}^{\infty} \frac{t^{n}}{n !}\left(\frac{\partial}{\partial u}\right)^{n}[F(x, u)]_{u=t_{1}} \\
& =\sum_{n=0}^{\infty} \frac{t^{n}}{n !}\left(\frac{\partial}{\partial u}\right)^{n}\left[x^{k} F(x, u)\right]_{u=t_{1}}
\end{aligned}
$$

Since $x^{k} F(x, t) \in H$, that is

$$
\Delta_{x}\left[x^{k} F(x, t)\right]=\frac{\partial}{\partial t}\left[x^{k} F(x, t)\right], \quad \Delta_{x} \equiv \frac{\partial^{2}}{\partial x^{2}}+\frac{2 v}{x} \frac{\partial}{\partial x}-\frac{\alpha^{2}}{x^{2}}
$$

Therefore from above,

$$
\begin{aligned}
x^{k} F\left(x, t+t_{1}\right) & =\sum_{n=0}^{\infty} \frac{t^{n}}{n !}\left(\Delta_{x}\right)^{n}\left[x^{k} F\left(x, t_{1}\right)\right] \\
& =e^{t \Delta x}\left[x^{k} F\left(x, t_{1}\right)\right] .
\end{aligned}
$$

The heat transform (3.4), can now be written, symbolically as,

$$
e^{t \Delta_{x}}\left[x^{k} F\left(x, t_{1}\right)\right]=x^{k} F\left(x, t+t_{1}\right)
$$

where

$$
e^{t \Delta x}[\phi(x)] \equiv \int_{0}^{\infty} U(\xi, x ; t) \phi(\xi) d \xi
$$


Therefore the inversion of (5.1) is then,

$$
x^{k} F\left(x, t_{1}\right)=e^{-t \Delta_{x}}\left[x^{k} F\left(x, t+t_{1}\right)\right],
$$

where from (3.6),

$$
e^{-t \Delta_{x}}[\psi(x)] \equiv \int_{0}^{i \infty} U(\xi, x ;-t) \psi(\xi) d \xi .
$$

In particular if we let $t_{1} \rightarrow 0$, then formally, (5.1) and (5.2) reduce to the pair

$$
e^{t \Delta x}\left[x^{k} f(x)\right]=x^{k} F(x, t)
$$

and

$$
x^{k} f(x)=e^{-t \Delta_{x}}\left[x^{k} F(x, t)\right],
$$

respectively, giving us a pair of heat transforms in operator form.

Example 1. Let

$$
f(x)=x^{\frac{1}{2}-k-v} I_{\mu}(x) .
$$

Then its heat transform is, [4, p. 197],

$$
F(x, t)=e^{t} x^{\frac{1}{2}-v-k} I_{\mu}(x), \quad \mu>-1 .
$$

Now,

$$
\begin{aligned}
e^{-t \Delta_{x}}\left[x^{k} F(x, t)\right] & =e^{-t \Delta_{x}}\left[e^{t} x^{\frac{1}{2}-v} I_{\mu}(x)\right] \\
& =e^{t} e^{-t \Delta_{x}}\left[x^{\frac{1}{2}-v} I_{\mu}(x)\right] \\
& =e^{t} \sum_{n=0}^{\infty} \frac{(-t)^{n}}{n !}\left(\Delta_{x}\right)^{n}\left[x^{\frac{1}{2}-v} I_{\mu}(x)\right] .
\end{aligned}
$$

But

$$
\Delta_{x}\left[x^{\frac{1}{2}-v} I_{\mu}(x)\right]=x^{\frac{1}{2}-v} I_{\mu}(x)
$$

hence,

$$
\left(\Delta_{x}\right)^{n}\left[x^{\frac{1}{2}-v} I_{\mu}(x)\right]=x^{\frac{1}{2}-v} I_{\mu}(x), \quad n=0,1,2, \ldots
$$

and

$$
\begin{aligned}
e^{-t \Delta_{x}}\left[x^{k} F(x, t)\right] & =e^{i} \sum_{n=0}^{\infty} \frac{(-t)^{n}}{n !} x^{\frac{1}{2}-v} I_{\mu}(x) \\
& =x^{\frac{1}{2}-v} I_{\mu}(x)=x^{k} f(x), \text { say, }
\end{aligned}
$$


where

$$
f(x)=x^{\frac{1}{2}-v-k} I_{\mu}(x)
$$

as predicted.

Example 2. Let

$$
F(x, t)=1
$$

Then

$$
x^{k} f(x)=e^{-\imath \Delta x}\left[x^{k}\right]=\sum_{n=0}^{\infty} \frac{(-t)^{n}}{n !}(\Delta x)^{n}\left[x^{k}\right]
$$

Now,

$$
\begin{aligned}
\Delta_{x}\left[x^{k}\right] & =\left(\frac{\partial^{2}}{\partial x^{2}}+\frac{2 v}{x} \frac{\partial}{\partial x}-\frac{\alpha^{2}}{x^{2}}\right)\left[x^{k}\right] \\
& =\left(k(k-1)+2 v k-\alpha^{2}\right) x^{k-2} \\
& =0
\end{aligned}
$$

since $k=\mu-v+\frac{1}{2}$ and $\mu^{2}=\left(v-\frac{1}{2}\right)^{2}+\alpha^{2}$. Therefore

$$
\left(\Delta_{x}\right)^{n}\left[x^{k}\right]=0, \quad n=1,2,3, \ldots
$$

and

$$
x^{k} f(x)=x^{k}
$$

so that

$$
f(x)=1
$$

Thus $f(x)=1$ and $F(x, t)=1$ gives us a pair of heat transforms, which can be verified by evaluating the integrals (3.7) and (3.8).

\section{Some special cases}

Let $\alpha=0$. The general heat equation (1.1) reduces to

$$
\frac{\partial^{2} u}{\partial x^{2}}+\frac{2 v}{x} \frac{\partial u}{\partial x}=\frac{\partial u}{\partial t}
$$

which has the solution, from (2.3), as

$$
U(\xi, x ; t)=\xi^{2 v} G_{v-\frac{1}{2}}(\xi, x ; t) .
$$


The heat transform (3.1), is then

$$
F(x, t)=\int_{0}^{\infty} \xi^{2 v} G_{v-\frac{1}{2}}(\xi, x ; t) f(\xi) d \xi,
$$

called the Poisson-Hankel transform, and its inversion is given by

$$
f(x)=\int_{0}^{\infty} \xi^{2 v} G_{v-t}(\xi, i x ; t) F(i \xi, t) d \xi,
$$

cf [4].

If we let $\alpha=\nu=0, \mu=-\frac{1}{2}$ then (1.1) reduces to

$$
\frac{\partial^{2} u}{\partial x^{2}}=\frac{\partial u}{\partial t}
$$

the ordinary heat equation, whose source solution is

$$
U(\xi, x ; t)=G_{-\frac{1}{2}}(\xi, x ; t)=\frac{1}{\sqrt{\pi t}} e^{-\left(\xi^{2}+x^{2}\right) / 4 t} \cosh \left(\frac{\xi x}{2 t}\right) .
$$

Also from (3.7) and (3.8), we have

$$
F(x, t)=\frac{1}{\sqrt{\pi t}} \int_{0}^{\infty} e^{-\left(\xi^{2}+x^{2}\right) / 4 t} \cosh \left(\frac{\xi x}{2 t}\right) f(\xi) d \xi
$$

and

$$
f(x)=\frac{1}{\sqrt{\pi t}} \int_{0}^{i \infty} e^{\left(\xi^{2}+x^{2}\right) / 4 t} \cosh \left(\frac{\xi x}{2 t}\right) F(\xi, t) d \xi .
$$

Symbolically, the operator

$$
\Delta_{x}=\frac{\partial^{2}}{\partial x^{2}}=D^{2},
$$

then (5.3) and (5.4) yield

$$
\begin{gathered}
F(x, t)=e^{t D^{2}}[f(x)] \\
f(x)=e^{-t D^{2}}[F(x, t)]
\end{gathered}
$$

which gives the Eddington solution of the ordinary heat equation, [5, p. 85]. 
Added in proof. The authors now realize that some of the results proved here may be deduced more simply from results in [1].

\section{REFERENCES}

1. D. T. Haimo and F. M. Cholewinski, The Weierstrass Hankel Convolution transform, $J$. d'Analyse Math. 17 (1966), 1-58.

2. ERdelyı et AL., Tables of Integral Transforms, Vol. I (McGraw-Hill Book Co., Toronto, 1954).

3. ERdelyi et AL., Tables of Integral Transforms, Vol. II (McGraw-Hill Book Co., Toronto, 1954).

4. D. T. Haimo and F. M. Cholewinski, Inversion of the reduced Poisson-Hankel transform, $J$. d'Analyse Math. 25 (1972), 323-343.

5. I. N. Sneddon, The Use of Integral Transforms (McGraw-Hill Book Co., Toronto. 1972).

Department of Mathematics

UNiversity OF CALGary

Calgary, Alberta

CANADA T2N 1N4 